\title{
High-Power Single-Mode Operation in DFB and FP Lasers Using Diffused Quantum-Well Structure
}

\author{
S. F. Yu, C. W. Lo, and E. Herbert Li, Senior Member, IEEE
}

\begin{abstract}
Distributed feedback (DFB) and Fabry-Perot (FP) semiconductor lasers with step and periodic interdiffusion quantum-well structures are proposed for high-power single-longitudinal-mode operation. It is shown that the phase-adjustment region formed by the diffusion step (i.e., step change in optical gain and refractive index) counteracts the influence of spatial hole burning, especially for DFB lasers with large coupling-length products biased at high injection current. Furthermore, it is found that with careful design of the diffusion grating (i.e., grating period and amount of diffusion extent) of FP lasers, side-mode suppression ratio can be enhanced and threshold current density can be minimized to a satisfied level.
\end{abstract}

Index Terms-Annealing, diffusion processes, distributed feedback lasers, Fabry-Perot resonators, laser modes, quantum wells, semiconductor device modeling, semiconductor lasers.

\section{INTRODUCTION}

$\mathbf{H}$ IGH-POWER AlGaAs-GaAs semiconductor lasers with stable single-longitudinal-mode operation are well suited for wavelength-selective applications such as frequency doubling and atomic spectroscopy [1]. Distributed feedback (DFB) lasers with $\lambda / 4$ phase-shifted are effective to provide singlelongitudinal-mode operation. However, stability is not maintained at high optical power especially for devices with large coupling-length products $(\kappa L>1.25)$ [2] due to nonuniform distribution of the refractive index which is a consequence of the longitudinal spatial hole burning (SHB) of carrier concentration [3]. Alternatively, new laser structures such as chirped gratings [4]-[6] or sampled gratings [7] are proposed to minimize the influence of longitudinal SHB in DFB lasers.

A simple fabrication technique and low production cost are the major advantages of Fabry-Perot (FP) lasers over other devices. However, the side-mode discrimination in an FP laser is poor especially for low-power operation or under direct electrical modulation. This is because the longitudinalmode discrimination is mainly determined by the material gain spectrum and is not affected by cavity loss or facet reflectivity [8]. Therefore, it is necessary to improve the side-mode suppression ratio (SMSR) without sacrificing the simple fabrication procedures of FP lasers. In this paper, we investigate the possibility of using a diffused quantum-well (DFQW) structure to improve the SMSR of DFB and FP semiconductor lasers.

A diffusion step along the longitudinal direction of the active region of the quantum wells (QW's) is proposed to

Manuscript received August 26, 1996; revised January 20, 1997.

The authors are with the Department of Electrical and Electronic Engineering, University of Hong Kong, Pokfulam Road, Hong Kong.

Publisher Item Identifier S 0018-9197(97)03803-7. enhance high-power stable longitudinal-mode operation of a uniform-grating DFB laser with large $\kappa L$. The operation principle of the DFQW DFB laser can be explained as follows.

1) The step DFQW's section provides a $\lambda / 4$ phase-shifted for single-longitudinal-mode oscillation.

2) Because the DFQW DFB laser has a uniform grating, the longitudinal SHB is less severe than the conventional $\lambda / 4$ DFB laser.

3) The step DFQW's profile compensates for any variation of refractive index arisen from longitudinal SHB of carrier concentration [2] and temperature effects [9] such that single-longitudinal-mode operation can be maintained at high power.

Therefore, significant reduction in SHB can be obtained by using a step DFQW structure.

A periodic DFQW structure is also proposed to improve the SMSR of FP semiconductor lasers. A periodic variation of refractive index and gain is created in the extent of interdiffusion along the longitudinal direction of the QW's active region which acts as a filter for the side modes. This DFQW FP laser is similar to a complex-coupled DFB laser with a highorder grating. The advantage of our proposed structure is that the complex grating can be in-phase or anti-phase; the choice depends on our selection of interdiffusion pattern and operating wavelength. However, we should avoid the following effects of DFQW grating in the design of FP lasers.

1) Higher order DFB modes can be excited by the diffusion grating provided that the grating period is much longer than the operating wavelength.

2) The optical gain of the QW's active layer (as well as the threshold current density) will reduce with the increase of diffusion extent.

Therefore, the period and extent of interdiffusion of the DFQW grating have to be determined for minimum number of DFB modes as well as threshold current density.

This paper is organized as follows. In Section II, the threshold and above threshold characteristics of DFB lasers with diffusion step structure are investigated. A self-consistent model of DFB lasers including the longitudinal variation of carrier concentration, photon density, refractive index, and temperature is utilized to calculate static and dynamic behavior of the proposed DFB laser. The electrical and optical properties of DFQW material are also described. In Section III, the threshold characteristics and design consideration of FP semiconductor lasers with periodic DFQW structure are presented. Finally, a brief discussion and conclusion are given in Section IV. 


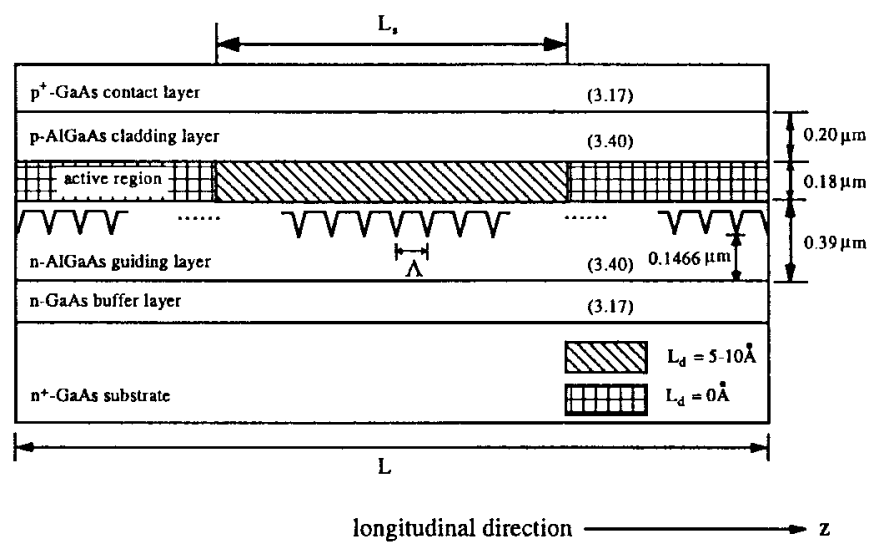

Fig. 1. Schematic of the DFB laser with diffusion step profile. $L_{s}$ is the length of the diffusion step and $L_{d}$ is the diffusion length.

\section{Proposed DFB LASERS With Diffusion SteP Profile}

\section{A. Laser Structure}

The schematic diagram of a DFB laser with diffusion step is shown in Fig. 1. The laser is composed of six layers: $n^{+}$ GaAs substrate, $\mathrm{n}-\mathrm{GaAs}$ buffer layer, $\mathrm{n}-\mathrm{AlGaAs}$ guiding layer in which the grating is defined, followed by a QW active layer, and finally the p-AlGaAs $(\cong 0.2 \mu \mathrm{m})$ and $\mathrm{p}^{+}$-GaAs $(\leq 0.3 \mu \mathrm{m})$ layers which form the cladder and contact, respectively. The active layer is regrown on top of the guiding layer which consists of four $\mathrm{Al}_{0.3} \mathrm{Ga}_{0.7} \mathrm{As}-\mathrm{GaAs}$ single $\mathrm{QW}$ 's with well and barrier thicknesses of 100 and $280 \AA$, respectively. It is proposed that a diffusion step is introduced in the center of the active region to form a phase-adjustment region (PAR) such that the optical gain and refractive index are slightly less than the as-grown region. In order to obtain a $\lambda / 4$ phaseshifted, the products of propagation coefficient difference and effective length of the PAR must be equal to $\pm\left(\frac{1}{2}+n\right) \pi$, where $n$ is a positive integer [2], [10]. The advantage of using an interdiffusion technique to form a PAR over 1) a phase-adjusted waveguide [2], [10] or 2) corrugation-pitch modulation [3] is that only simple fabrication procedures are required (i.e., DFQW structure can be easily obtained by impurities implantation and thermal annealing).

\section{B. Models for DFB Lasers and QW Material}

The propagation of forward and reverse fields, $F$ and $R$, along the laser cavity can be described by the time-dependent coupled optical wave equations given as follows [11]:

$$
\begin{aligned}
\left(\frac{1}{\nu_{g}} \frac{\partial}{\partial t} \pm \frac{\partial}{\partial z}\right)\left[\begin{array}{l}
F \\
R
\end{array}\right]= & {\left[\frac{1}{2}\left(\Gamma G-\alpha_{s}\right)+j \delta \beta\right]\left[\begin{array}{l}
F \\
R
\end{array}\right] } \\
& +j \kappa\left[\begin{array}{l}
R \\
F
\end{array}\right]+\left[\begin{array}{l}
j_{F s} \\
j_{R s}
\end{array}\right]
\end{aligned}
$$

where $j=\sqrt{-1}, \kappa$ is the coupling coefficient, $G$ is the modal gain, $\alpha_{s}$ is the absorption and scattering loss of the $\mathrm{QW}$ waveguide, $j_{F s, R s}$ is the spontaneous emission, $\nu_{g}\left(=c / n_{g}\right.$, where $n_{g}$ is group index and $c$ is the velocity of light in free space) is the group velocity, and $\Gamma$ is the transverse optical confinement factor. The deviation from Bragg's condition, $\delta \beta$, is given as

$$
\delta \beta=\frac{\omega_{o}}{c}(n e+\Delta n \Gamma)-\frac{\pi}{\Lambda}
$$

where $\omega_{o}\left(=2 \pi c / \lambda_{o}\right)$ is the lasing frequency, $\lambda_{o}$ is the operating wavelength, and $\Lambda$ is the period of grating. ne is the effective refractive index of the grating waveguide and $\Delta n$ is the change of refractive index due to the variation of carrier concentration. ne can be evaluated by the effective index method provided that the refractive index profile of the laser is known. The time-dependent rate equation of carrier concentration along the longitudinal direction of the active region is described by

$$
\frac{\partial N}{\partial t}=\frac{J}{q N_{W} L_{z}}-\frac{N}{\tau_{N}}-\nu_{g} G P
$$

where $J$ is the current density, $N_{w}$ is the number of QW's, $L_{z}$ is the thickness of the $\mathrm{QW}, q$ is the electron charge, $P\left(=|F|^{2}+|R|^{2}\right)$ is the photon density, and $\tau_{N}$ is the carrier lifetime. In the model, the heat distribution along the longitudinal active region is also taken into account by solving the time-dependent quasi-two-dimensional heat equation (see Appendix A). As a result, threshold and above-threshold behavior of DFB semiconductor lasers with DFQW structure can be obtained by solving (1), (3), and the heat equation in a self-consistent manner [11].

The refractive index and optical gain of QW material under the influence of impurities-induced compositional disordering are also considered in our analysis. The models given in [12]-[14] are utilized to calculate the optical and electrical properties of DFQW's which are summarized in Appendix B. It is defined that the extent of diffusion into the $\mathrm{QW}$ material is characterized by a diffusion length, $L_{d}\left[=\sqrt{\left(D \tau_{a}\right)}\right]$ where $\tau_{a}$ is the annealing time and $D$ is the temperature-dependent diffusion coefficient [15]. It is assumed that $L_{d}=0 \AA$ represents the as-grown QW's and the diffusion extent is described by the magnitude of $L_{d}$. Fig. 2 shows the influence of $L_{d}$ on the optical gain and refractive index spectrum (TE polarization) of the QW material (at $300 \mathrm{~K}$ ). It is observed, for $\lambda_{\circ} \geq 0.85 \mu \mathrm{m}$, the optical gain as well as the refractive index are reduced with $L_{d}$. In the following calculations, it is assumed that the lasers have perfect antireflection coating on both facets. Furthermore, the parameters used in the calculation are given in Tables I-III.

\section{Threshold Characteristics of Diffusion-Step DFB lasers}

Fig. 3 shows the gain margin and the detuned wavelength of the gap mode [16] $\delta \lambda\left(=-\delta \beta \lambda_{o}^{2} / 2 \pi n_{g}\right)$ against the effective length $L_{s}$ of PAR for $L_{d}$ equal to 5 and $10 \AA$. It is assumed that $\kappa L$ varies between 1.25 and 2.8. For a device with $L_{d}=$ $5 \AA$ [see Fig. 3(a)], it is observed that the gain margin has a peak for $L_{s}$ varies between 120 and $160 \mu \mathrm{m}$. The wavelength of the gap mode decreases monotonically with $L_{s}$ due to the reduction of effective refractive index along the cavity. For $L_{d}=10 \AA$ [see Fig. 3(b)], the devices exhibit similar characteristics to $L_{d}=5 \AA$. However, the range of $L_{s}$ for peak gain margin is reduced with the increase of $L_{d}$ due to the reduction of refractive index and optical gain inside the PAR. It must be noted that the peak gain margin of the conventional 
TABLE I

PARAmeters USED IN THE Model

\begin{tabular}{c|c}
\hline Parameters (symbol) & Magnitude \\
\hline \hline Operating wavelength $\left(\lambda_{\mathrm{o}}\right)$ & $0.85 \mu \mathrm{m}$ \\
\hline Grating Period $(\Lambda)$ & $0.127 \mu \mathrm{m}$ \\
\hline Absorption and scattering loss in waveguide $\left(\alpha_{\mathrm{s}}\right)$ & $40 \mathrm{~cm}^{-1}$ \\
\hline Width of active layer $(\mathrm{w})$ & $2.0 \mu \mathrm{m}$ \\
\hline Total thickness of active layer $\left(\mathrm{t}_{\mathrm{d}}\right)$ & $0.18 \mu \mathrm{m}$ \\
\hline Thickness of the quantum well $\left(\mathrm{L}_{\mathrm{z}}\right)$ & $100 \AA$ \\
\hline Number of quantum well $\left(\mathrm{N}_{\mathrm{w}}\right)$ & 4 \\
\hline Carrier lifetime $\left(\tau_{\mathrm{N}}\right)$ & $3 \mathrm{~ns}$ \\
\hline Effective group refractive index $\left(\mathrm{n}_{\mathrm{b}}\right)$ & 3.70 \\
\hline Length of laser cavity $(\mathrm{L})$ & $400 \mu \mathrm{m}$ \\
\hline Velocity of light in free space $(\mathrm{c})$ & $3 \times 10^{10} \mathrm{cms}^{-1}$ \\
\hline
\end{tabular}

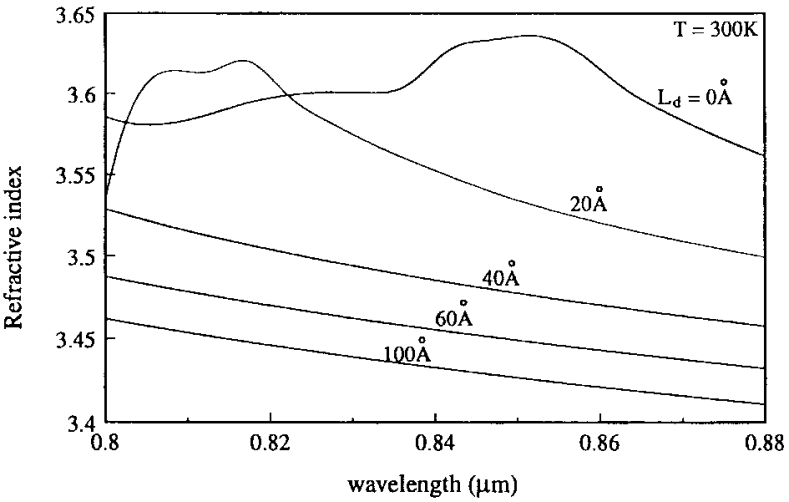

(a)

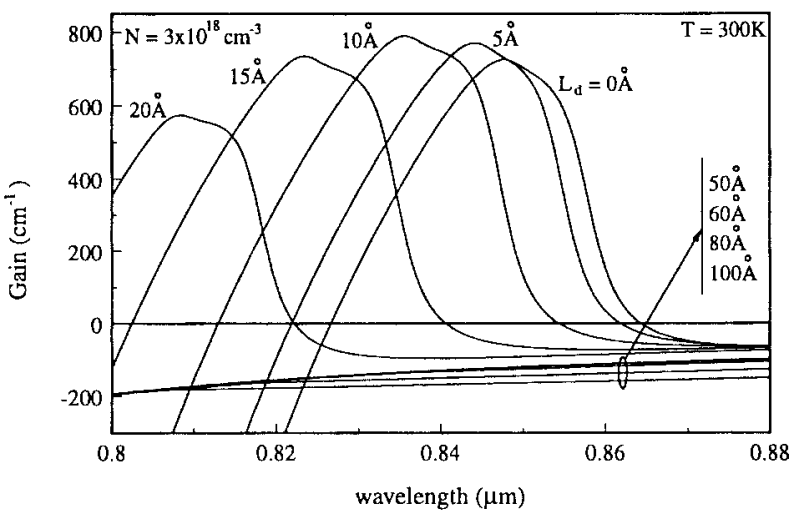

(b)

Fig. 2. Calculated (a) background refractive index and (b) optical gain spectra of $\mathrm{Al}_{0.3} \mathrm{Ga}_{0.7} \mathrm{As}-\mathrm{GaAs} \mathrm{QW}$ at carrier concentration, $N=3 \times 10^{18}$ $\mathrm{cm}^{-3}$ with various levels of $L_{d}$.

phase-adjusted waveguide devices [2], [10] is independent of the length of PAR.

\section{Static and Dynamic Characteristics of Diffusion-Step DFB Lasers}

Fig. 4 compares the variation of SMSR with normalized injected current density, $J / J_{\text {th }}$ (where $J_{\text {th }}$ is the threshold current density) at steady state for the lasers with $\left(L_{d}=\right.$ $5 \AA$ ) and without (conventional discrete $\lambda / 4$ DFB laser) step diffusion profile. Multimode operation (defined by a drop of SMSR from 50 to $10=40 \mathrm{~dB}$ ) is observed for conventional $\lambda / 4$ DFB laser with $\kappa L \geq 3$.2. However, stable

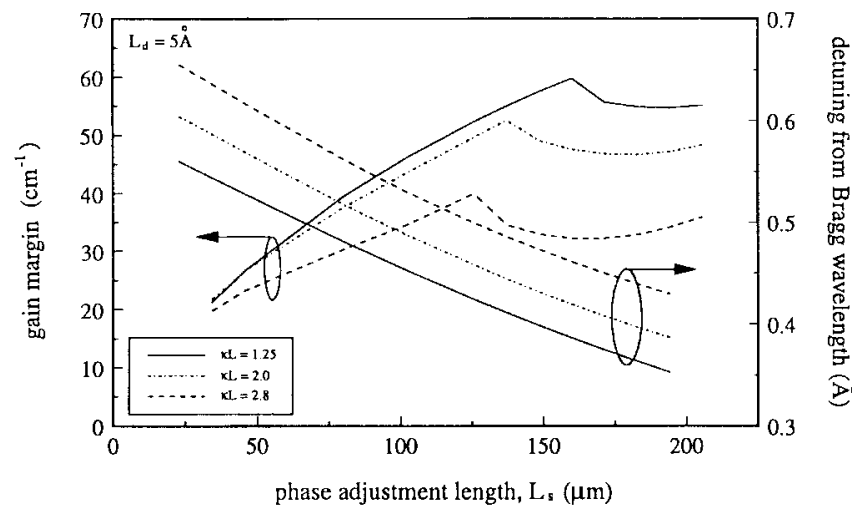

(a)

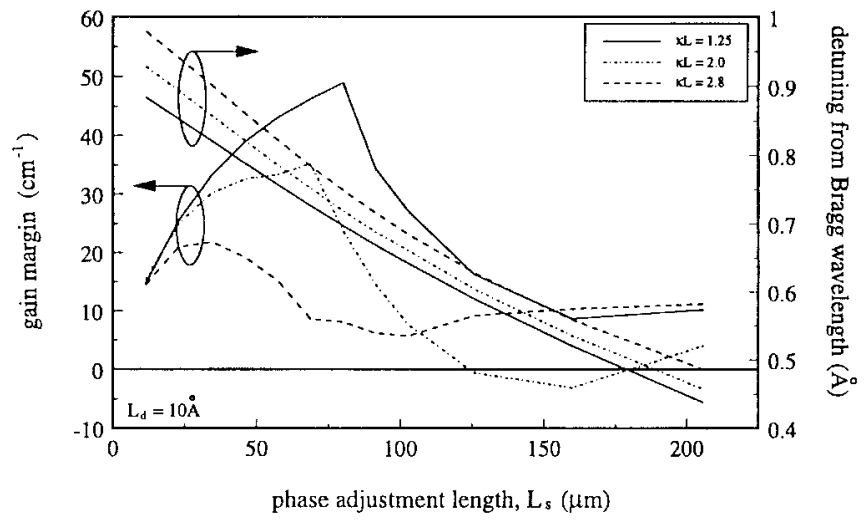

(b)

Fig. 3. The variation of gain margin and detuning wavelength of gap mode against $L_{s}$ for devices with $\kappa L=1.25,2.0$, and 2.8. It is assumed that (a) $L_{d}=5 \AA$ and (b) $L_{d}=10 \AA$.

single longitudinal mode is maintained for laser with step diffusion profile. This is because of the built-in step refractive index profile against the carrier-induced index change inside the active region. The maximum output power of the lasers is larger than $50 \mathrm{~mW}$ at $J / J_{\mathrm{th}}=10$. For the device with $\left(L_{d}=10 \AA\right)$, similar behavior is observed and hence it is not described again.

The relative effective refractive index profiles of devices with $\kappa L=3.2$ and biased at $J=9 J_{\text {th }}$ are shown in Fig. 5 . As we can see, the conventional $\lambda / 4$ DFB laser exhibits nonuniform (concave-up) distribution of refractive index with peak to peak value, $\Delta n_{p}$, equal to 0.0015 . However, the 
TABLE II

Material Parameters in the Laser Structure

\begin{tabular}{|c|c|c|c|c|}
\hline \multirow[b]{2}{*}{$\begin{array}{c}\text { (at operating wavelength of } \\
0.85 \mu \mathrm{m} \text { ) }\end{array}$} & \multicolumn{4}{|c|}{ Diffusion Length $\left(\mathrm{L}_{\mathrm{d}}\right)$} \\
\hline & $\overline{0 A \AA}$ & $\overline{5 \AA}$ & $10 \AA$ & $20 \AA$ \\
\hline Fitted parameter $\left(a_{0}\right) \mathrm{cm}^{-1}$ & 1591.6434 & 1384.6995 & 432.1735 & 65.5869 \\
\hline $\begin{array}{l}\text { Transparency carrier density } \\
\left(\mathrm{N}_{\mathrm{o}}\right) \times 10^{18} \mathrm{~cm}^{-3}\end{array}$ & 1.9399 & 1.8587 & 2.2196 & 11.7549 \\
\hline Fitted parameter $\left(\mathrm{d}_{\mathrm{o}}\right)$ & -0.02830 & -0.02783 & -0.02644 & -0.02539 \\
\hline Fitted parameter $\left(\mathrm{N}_{\mathrm{r}}\right) \times 10^{18} \mathrm{~cm}^{-3}$ & 2.0557 & 1.9771 & 2.4109 & 11.7883 \\
\hline Refractive index $\left(n_{r}\right)$ & 3.6270 & 3.6074 & 3.5880 & 3.5360 \\
\hline Effective refractive index (ne) & 3.302938 & 3.301600 & 3.300346 & 3.296800 \\
\hline
\end{tabular}

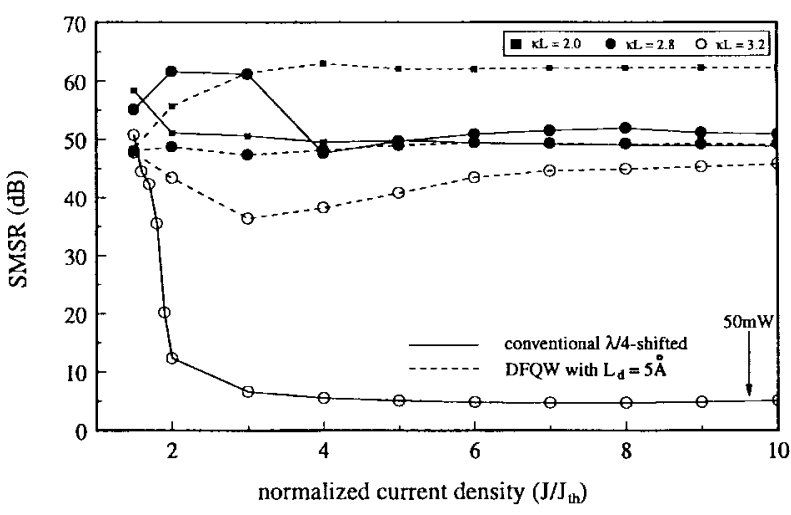

Fig. 4. The SMSR various with normalized current, $J / J_{\text {th }}$, for lasers with $\kappa L=2.0(\square), \kappa L=2.8(\bullet)$, and $\kappa L=3.2(0)$. The solid and dash lines represent the cases for the conventional $\lambda / 4$ phase-shifted DFB laser and step-diffused device $\left(L_{d}=5 \AA\right)$.

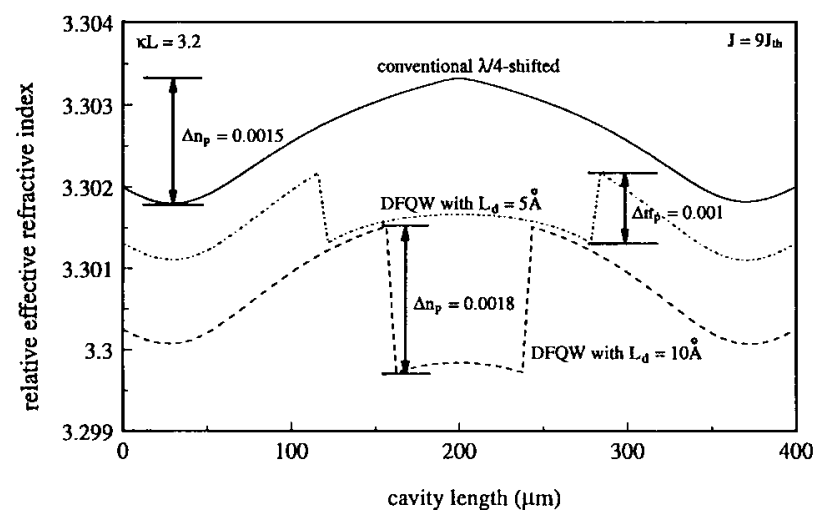

Fig. 5. Longitudinal refractive index profile of the conventional $\lambda / 4$ DFB laser (solid line), DFQW laser with $L_{d}=5 \AA$ (dashed-dotted line) and DFQW laser with $L_{d}=10 \AA$ (dashed line). The devices are biased at $J=9 J_{\text {th }}$ with $\kappa L$ equal to 3.2 .

uniformity of the effective refractive index is maintained for $L_{d}=5 \AA$ with $\Delta n_{p}=0.001$. For $L_{d}=10 \AA$, the built-in refractive index step is larger than the required to overcome the SHB effects and $\Delta n_{p}$ is found to be equal to 0.0018 . Therefore, only a small range of $L_{d}\left(5 \AA \leq L_{d}<10 \AA\right)$ will satisfy the requirement to minimize the influence of SHB effects. It must be noted that device with $L_{d}<5 \AA$ is not realistic due to the limitation of controlled interdiffusion.

The spectrum purity of the turn-on transient signal determines the maximum modulation bandwidth of the lasers. In our purposed DFB lasers, a built-in step refractive index profile is

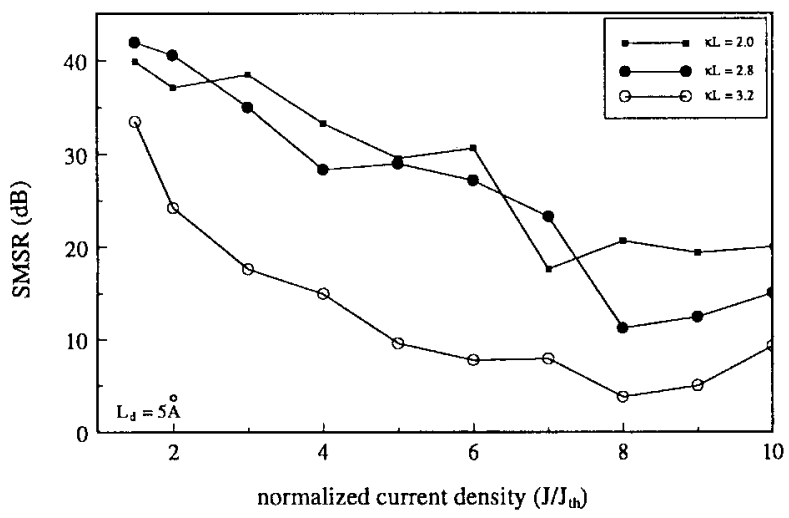

(a)

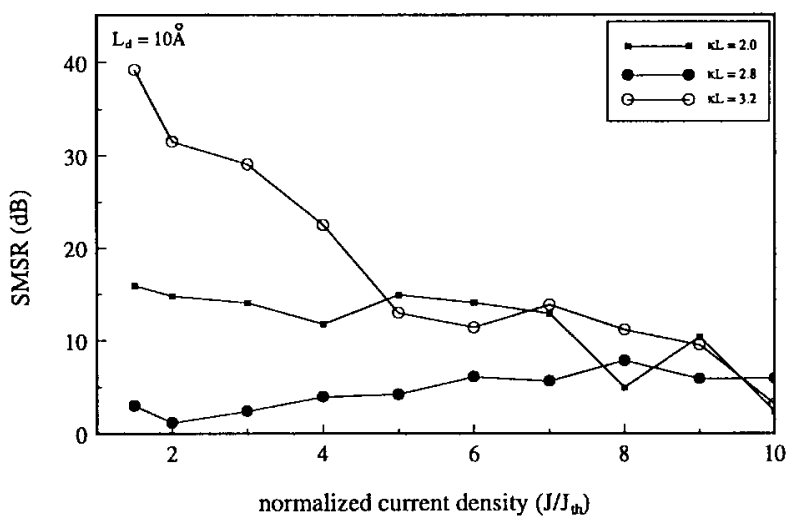

(b)

Fig. 6. The SMSR of the first overshoot power spectrum various with normalized current, $J / J_{\mathrm{th}}$, for a device with diffusion length (a) $L_{d}=5$ $\AA$ and (b) $L_{d}=10 \AA$. The symbols $(\boldsymbol{\bullet}),(\bullet)$, and (o) represent $\kappa L=2.0$, 2.8 , and 3.2 , respectively.

introduced (by interdiffusion) which may affect the spectrum purity of the lasers as SHB is negligible during the turn-on time interval. Fig. 6 shows the influence of built-in index step on the SMSR at the first overshoot of lasers with $L_{d}=5$ and $10 \AA$. As we can see, SMSR is reduced with the increase of injection current and devices with low $\kappa L$ exhibit better SMSR. The reduction of SMSR can be attributed to the builtin index profile because no SHB is taken place during the first overshoot of the output power. The built-in refractive index step reduces the gain requirement of the band-edge mode. Therefore, excitation of band-edge mode is observed in both cases and the case $L_{d}=10 \AA$ is more pronounced than $L_{d}=$ $5 \AA$. 


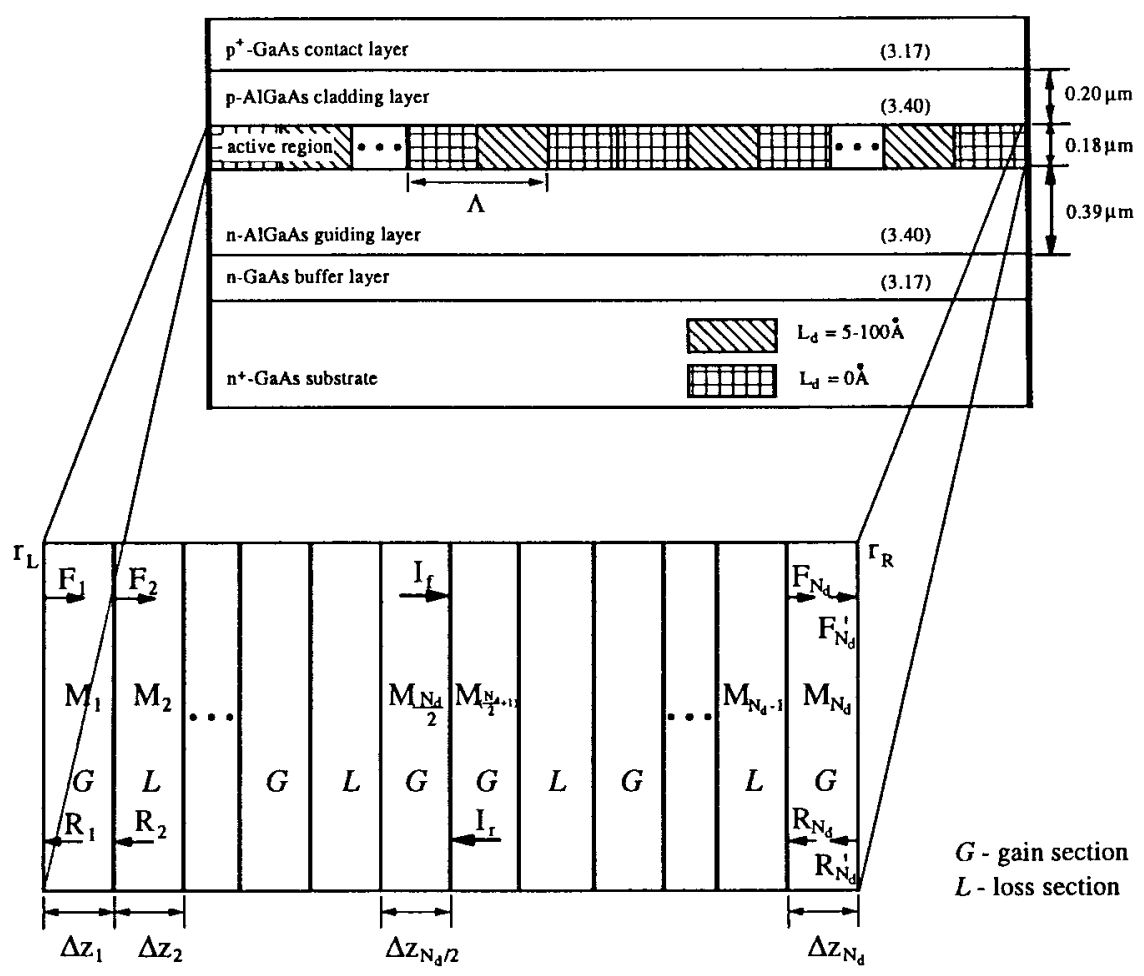

(a)

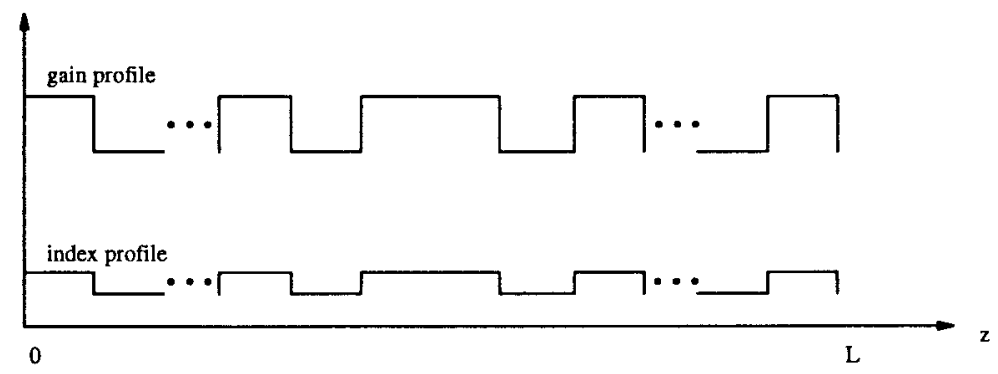

(b)

Fig. 7. (a) The schematic of an FP laser with periodic DFQW structure. (b) The periodic variation of gain and built-in refractive index profile along the $\mathrm{QW}$ active region is due to the periodic change of diffusion length.

\section{PRoposed FP LASERS WITH PERIODIC DFQW STRUCTURE}

\section{A. Laser Structure}

Fig. 7 shows the schematic diagram of a FP laser with periodic DFQW structure. The device is similar to that given in Fig. 1 except no grating is introduced. The periodic variation of gain and refractive index (along the longitudinal direction of active region) is obtained by periodic interdiffusion. The as-grown QW section $\left(L_{d}=0 \AA\right)$ serves as gain region while the diffused section $\left(L_{d}=5-100 \AA\right)$ serves as the loss region with large differences of refractive index as well as optical gain. The longitudinal length of each diffused section, $\Delta z_{k}$ (for $k=1,2, \cdots, N_{d}$ ), is equal to a multiple of $\lambda_{o} / 4$, where $N_{d}$ is the total number of diffused sections. The device's total length is set to $400 \mu \mathrm{m}$ with $N_{d}$ varying between 8 and 160 , which can be done by alternating the period of the diffusion grating. The left and right facet reflectivities of the laser cavity are both assumed to be 0.55 .

\section{B. Model for FP Lasers with Periodic DFQW Structure}

The optical fields propagating along the diffusion grating can be calculated by using the transfer matrix method [17]. In each diffused section, carrier density, photon density, refractive index, and other material parameters are assumed to be constants but all these parameters can be varied along the cavity. The spontaneous emission can also be taken into consideration by introducing between sections [18]. Detailed modeling of optical fields inside the active region can be found in Appendix C. The rate equation of carrier concentration along the DFQW's active region is described by

$$
\begin{aligned}
\frac{d N_{k}}{d t}= & \frac{J}{q N_{w} L_{z}}-\frac{N_{k}}{\tau_{N}} \\
& -\nu_{g} \sum_{m} \Gamma_{k} G_{k}\left(\lambda_{m}\right) P_{k}\left(\lambda_{m}\right)
\end{aligned}
$$

where $k$ is the section number, $\lambda_{m}$ is the mode wavelength, $P_{k}$ is the photon density, and $\Gamma_{k}$ is the transverse optical confinement factor of the $k$ th section. The below and above 


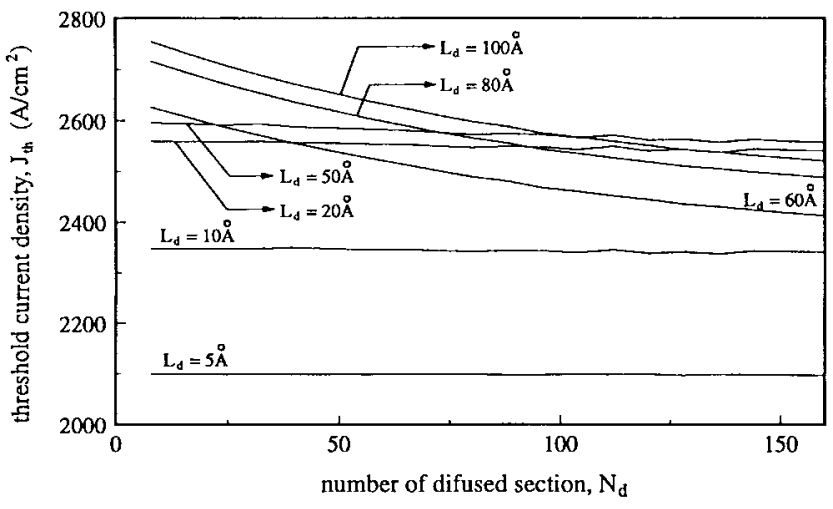

Fig. 8. The threshold current density against total number of diffused sections with the gain region is defined by diffusion length, $L_{d}=0 \AA$, and $L_{d}$ in the loss region is set to $5,10,20,50,60,80$, and $100 \AA$, respectively.

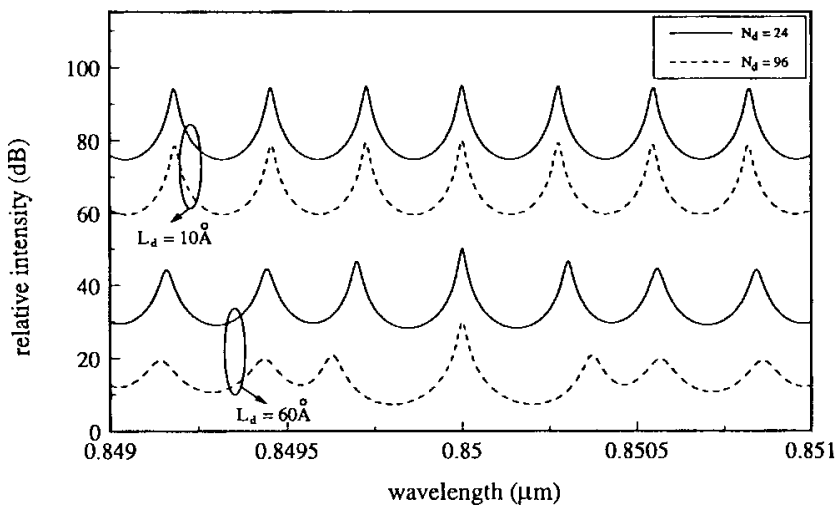

Fig. 9. Threshold emission spectra of DFQW's FP lasers for $L_{d}=10 \AA$ and $N_{d}=24, L_{d}=10 \AA$ and $N_{d}=96, L_{d}=60 \AA$ and $N_{d}=24$, and $L_{d}=60 \AA$ and $N_{d}=96$.

threshold optical spectral can be obtained by solving the transfer matrix equation, carrier rate equation, and heat equation self-consistently.

\section{Below and Above Threshold Characteristics of DFQW FP Lasers}

Fig. 8 shows a plot of the threshold current density, $J_{\text {th }}$, of the periodic DFQW's FP laser against the total number of diffused sections, $N_{d}$, with $L_{d}$ as a variable parameter. It is found that for large $L_{d}(>50 \AA), J_{\text {th }}$ is inversely proportional to $N_{d}$. In addition, a maximum $J_{\text {th }}$ is located at $L_{d}$ 's combination approximately equal to $0 \mid 100 \AA$ for $N_{d}<80$ and equal to $0 \mid 50 \AA$ for $N_{d}>80$. This is because the optical distributed feedback is affected by the design of the periodic DFQW structure. It is noted that optical gain at $0.85 \mu \mathrm{m}$ can only be obtained by external carrier injection for DFQW's with $L_{d}<15 \AA$ [see Fig. 2(b)]. For $L_{d}>60 \AA$, optical feedback is enhanced due to the large difference in gain and refractive index between grating sections (see Fig. 2). Therefore, low threshold current density can be obtained at $L_{d}<10 \AA$ or $L_{d}>60 \AA$ and is also a function of $N_{d}$. It must be noted that the threshold current density of FP lasers without periodic DFQW structure is equal to $2123 \mathrm{~A} / \mathrm{cm}^{2}$. Fig. 9 shows the corresponding threshold-amplified spontaneous spectra for

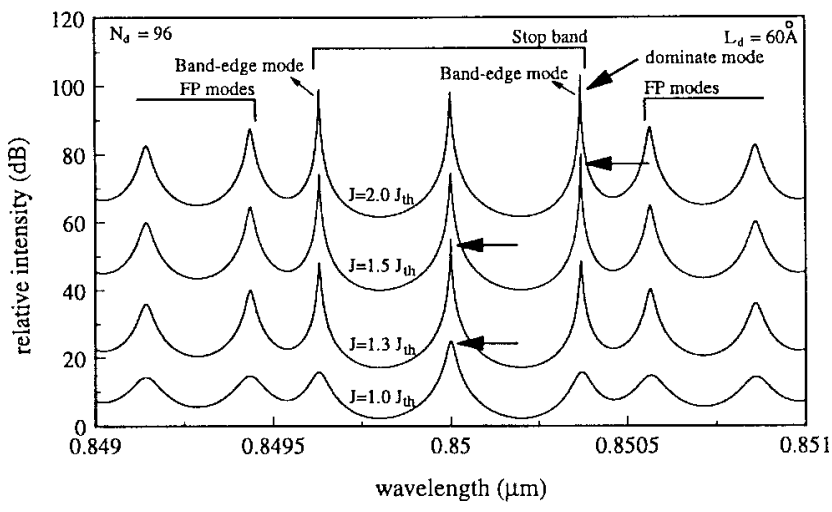

(a)

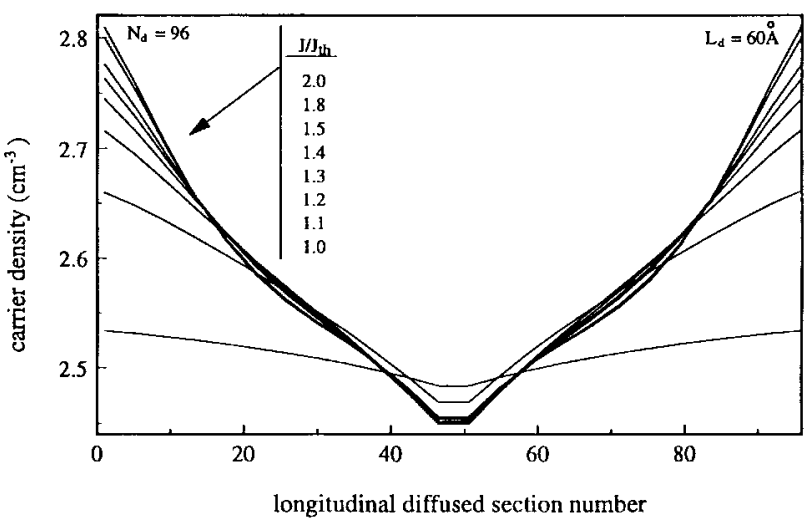

(b)

Fig. 10. (a) The spontaneous emission power spectra of DFQW FP lasers. The device has 96 gain/loss diffused sections and $L_{d}$ 's combination is equal to $0 \mid 60 \AA$. The injection current density is set to $1.0 J_{\mathrm{th}}, 1.3 J_{\mathrm{th}}, 1.5 J_{\mathrm{th}}$, and $2.0 J_{\mathrm{th}}$. (b) The carrier concentration profile of the lasers with injection current density set to $1.0 J_{\mathrm{th}}, 1.1 J_{\mathrm{th}}, 1.2 J_{\mathrm{th}}, 1.3 J_{\mathrm{th}}, 1.4 J_{\mathrm{th}}, 1.5 J_{\mathrm{th}}, 1.8 J_{\mathrm{th}}$, and $2.0 J_{\mathrm{th}}$.

$L_{d}$ 's combination is equal to $0 \mid 10$ and $0 \mid 60 \AA$ (with $N_{d}$ equal to 24 and 96, respectively). As shown in the figure, the bandgap mode is dominant in the spectra especially for $L_{d}$ 's combination equal to $0 \mid 60 \AA$ and $N_{d}=96$. This is expected as the filtering properties of the periodic DFQW structure are more efficient for large magnitude of $L_{d}$ and $N_{d}$. In the design of devices with DFQW's, the value of $L_{d}$ should not be greater than $100 \AA$; otherwise the electrical and optical properties of QW's will be removed.

Fig. 10(a) shows the amplified spontaneous spectra for device with $L_{d}$ 's combination equal to $0 \mid 60 \AA$ and $N_{d}=96$. As we can see, when the current density increases from $1.0 J_{\text {th }}$ to $1.3 J_{\mathrm{th}}$, the bandgap mode dominates over other longitudinal modes. Further increase in current density excites the bandedge mode (with longer wavelength) due to the SHB effects. Fig. 10(b) shows the corresponding longitudinal distribution of carrier concentration at different injection levels. At the injection level equal to $1.5 J_{\mathrm{th}}$, the longitudinal carrier distribution changes rapidly due to the excitation of band-edge mode but it is stabilized with further increase in injection current.

As we have mentioned before, because the period of the diffusion grating is in the order of $5 \mu \mathrm{m}$, it is expected that the DFB modes are repeated in the optical spectrum. In order to avoid the influence of the high-order DFB modes, the bandgap 
mode should be selected in coherent with the optical gain peak of the active region $\left(L_{d}=0 \AA\right)$ such that other DFB modes away from the peak gain wavelength are suppressed. In the above calculations, it is assumed that the wavelength of the bandgap mode (i.e., $\lambda_{o}=0.85 \mu \mathrm{m}$ ) is coherent with the optical gain peak (QW's active region with $L_{d}=0 \AA$ ).

\section{DISCUSSION}

The success of our proposal depends upon whether we can realize the DFQW semiconductor lasers in practice using existing fabrication technologies such that the production cost and waste in the device's fabrication can be further reduced. The following must be noted.

1) The proposed semiconductor lasers have typical dimensions which require a simple processing technique and are compatible with existing fabrication technologies.

2) Interdiffusion of QW's requires the penetration of impurities or vacancies through the contact and cladding layers into the active region such that the contact and cladding layers form a blocking layer of the diffusion process. However, the total thickness of the $\mathrm{p}^{+}{ }_{-} \mathrm{GaAs}$ contact layer and the p-AlGaAs cladding layer is less than $1 \mu \mathrm{m}$, which allows the diffusion process to be carried out [19].

3) The diffusion length $L_{d}$ of DFQW's active region is determined by the implantation energy and thermal annealing time of the interdiffusion process. With careful control of annealing temperature and time, $L_{d}$ down to $5 \AA$ can be obtained without any difficulty.

4) The formation accuracy of DFQW's grating determines the yield rate of single-longitudinal-mode operation of the FP lasers. The combined technologies of electron beam lithography and implantation-enhanced intermixing [20] are utilized to realize a structure which is far more precise than the required micron DFQW grating.

In fact, the use of DFQW's structure to improve singlelongitudinal-mode operation of $\lambda / 4$ DFB lasers has been proposed [21] and gain-coupled DFB lasers with periodic DFQW structure have also been fabricated [22]. These indicated that our proposed DFQW structures can easily be realized in practice. The other advantages for adopting DFQW structure are: 1) tunability of the operating wavelength by modifying the diffusion profile; 2 ) the use of the interdiffusion technique to form a PAR in DFB lasers can avoid the complex fabrication process in $\lambda / 4$ phase-shifted corrugation; and 3) enhanced yield rate of single-longitudinal-mode operation of uniform grating DFB lasers and FP lasers.

\section{CONCLUSION}

We have proposed novel structures for DFB and FP semiconductor lasers by interdiffusion of a $\mathrm{QW}$ active region. It is shown that stable single-longitudinal-mode operation can be maintained in DFB lasers with uniform grating at high output power. For DFB lasers with large $\kappa L$, the influence of SHB at steady state can be minimized (i.e., enhancement of SMSR) with diffusion step of $L_{d} \geq 5 \AA$ and $L_{d}<10 \AA$. However, it is shown that SMSR is deteriorated by the diffusion step

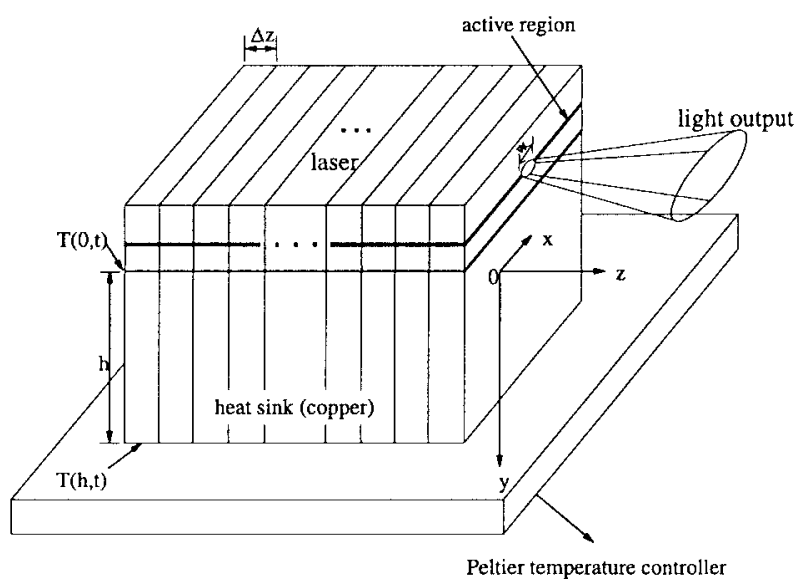

Fig. 11. Laser-mounting structure with a copper heat sink.

structure during the turn-on time interval especially for $L_{d}>5$ $\AA$. Single-longitudinal-mode operation is also achieved in DFQW FP lasers at and above threshold. It is shown that the DFQW structure also enhances the SMSR of FP lasers. This is because of the filtering effects arise from the difference of refractive index and optical gain between the diffused sections. It is revealed that a device with $L_{d}$ 's combination equal to $0 \mid 60 \AA$ exhibits better spectrum purity than a device with $L_{d}$ 's combination equal to $0 \mid 10 \AA$; even the threshold current densities of both devices are close together. This is because the periodic DFQW structure demonstrates better optical filtering efficiency with large $L_{d}$ which compensates for the increase of optical loss arisen from the interdiffusion effects. Although the threshold current density of the proposed FP lasers with DFQW's structure is deteriorated by 16\% (compares with FP lasers without interdiffusion structure), the SMSR is enhanced by more than $10 \mathrm{~dB}$ at threshold.

\section{APPENDIX A}

In order to estimate the heat distribution along the active region, several assumptions are made.

1) The longitudinal heat distribution is analyzed by dividing the laser cavity/copper heat sink into small segments (see Fig. 11).

2) In each segment, the transverse heat flow is described by the one-dimensional time-dependent Poisson equation [23]

$$
\kappa_{p} \frac{\partial^{2} T(y, t)}{\partial y^{2}}=\rho C_{p} \frac{\partial T(y, t)}{\partial t}
$$

where $T$ is the temperature measured in Kelvin, $\rho$ is the density, $C_{p}$ is its specific heat, and $\kappa_{p}$ is its thermal conductivity of the heat sink. $y>0$ corresponds to the copper of heat sink and $y<0$ to all semiconductor layers.

3) Although the heat distribution is much pronounced in the lateral direction ( $x y$ plane), we ignore the influence of thermal effects along the lateral direction. This is because we concentrate on the analysis of longitudinal modes operation. Therefore, we assume the fundamental lateral mode is maintained at a high power such that the term $\partial^{2} T / \partial x^{2}$ is ignored in (A1). Furthermore, the 
lateral dimension of the laser and copper heat sink are assumed identical in order to reduce our computational effort.

4) The longitudinal thermal diffusion length is assumed to be much less than the segment length such that the longitudinal term $\partial^{2} T / \partial z^{2}$ can be ignored in (A1).

5) The corresponding boundary conditions of (A1) are given by

$$
T(h, t)=T_{p}
$$

and

$$
T(0, t)=H(t) \frac{w d}{4 \kappa_{p}}
$$

where $w$ is the width, $d$ is the thickness of the active region, $h$ is the height of the heat sink, and $T_{p}$ is the temperature of electrical Peltier temperature controller. $H(t)$ is the power dissipated along the active region and can be approximated by

$$
H(t)=\frac{N_{w} V_{d}(N) J}{L_{z}}-\frac{\nu_{g} \hbar \omega P}{\Delta z}
$$

where $V_{d}$ is the junction voltage, $N_{w}$ is the number of quantum wells, $L_{z}$ is the width of wells, and $N$ is the carrier concentration inside the active region. $V_{d}$ can be approximated by [24]

$$
\begin{aligned}
V_{d}(N)=\frac{1}{q} & \left(E_{g}+k_{B} T\right. \\
\cdot & \left.\ln \left\{\left[\exp \left(\frac{N}{N_{c}}\right)-1\right]\left[\exp \left(\frac{N}{N_{V}}\right)-1\right]\right\}\right)
\end{aligned}
$$

where $E_{g}$ is the energy gap between the first quantized energy level of conduction and valence bands of the QW, $k_{B}$ is the Boltzmann constant, and $T$ is the temperature in Kelvin. $N_{C}$ and $N_{V}$ are the effective conduction and valence edge density of states, respectively. They can be expressed as $N_{c / V}=m_{e / h}^{*} k_{B} T / \pi \hbar^{2} L_{z}$ where $m_{e / h}^{*}$ is the effective mass of the electron/hole.

6) The time variation of temperature can be approximated by

$$
\left.\frac{\partial T}{\partial t}\right|_{y_{j}} \Delta t=T\left(y_{j}, t+\Delta t\right)-T\left(y_{j}, t\right)
$$

where $j$ is an integer, $\Delta t$ is identical to that given in the wave equations, and the time variation of the temperature is synchronized with the traveling waves and carrier concentration. Substituting (A5) into (A1), the rate equation of temperature can be written as

$T\left(y_{j}, t+\Delta t\right)-T\left(y_{j}, t\right)=\frac{1}{\rho C_{p}}\left\{\left.\kappa_{p} \frac{\partial^{2} T(y, t)}{\partial y^{2}}\right|_{y_{j}}\right\} \Delta t$

where

$$
\left.\frac{\partial^{2} T(y, t)}{\partial y^{2}}\right|_{y_{j}}=\frac{T\left(y_{j+1}, t\right)-2 T\left(y_{j}, t\right)+T\left(y_{j-1}, t\right)}{\Delta y^{2}}
$$

for $y_{i}$ away for the facets of device. At the boundaries, the corresponding second derivative takes the form

$$
\begin{aligned}
& \left.\frac{\partial^{2} T(y, t)}{\partial y^{2}}\right|_{y_{1}}=\frac{2\left[T\left(y_{2}, t\right)-\frac{H(t) d w}{2 \sigma}\right]}{\Delta y^{2}} \\
& \left.\frac{\partial^{2} T(y, t)}{\partial y^{2}}\right|_{y_{n}}=-\frac{2\left[T_{p}-T\left(y_{n-1}, t\right)\right]}{\Delta y^{2}}
\end{aligned}
$$

where $n$ is the total number of section, $y_{1}=0$, and $y_{n}=h$. In the calculation, it is assumed that $\kappa_{p}=4$ $\mathrm{W} \cdot \mathrm{m}^{-1} \cdot \mathrm{K}^{-1}, E_{g}=1.519-5.408 \times 10^{-4} \times T^{2} /(T+$ 204) $\mathrm{eV}, h=1 \mathrm{~mm}$, and $T_{p}=300 \mathrm{~K}$.

\section{APPENDIX B}

The refractive index, $n_{\mathrm{DFQW}}$, of the DFQW's active layer is given by [12]

$$
n_{\mathrm{DFQW}}(\omega)=\left(\frac{1}{2} \varepsilon_{1}^{T}(\omega)+\frac{1}{2}\left\{\left[\varepsilon_{1}^{T}(\omega)\right]^{2}+\left[\varepsilon_{2}^{\Gamma}(\omega)\right]^{2}\right\}^{1 / 2}\right)^{1 / 2}
$$

where $\omega$ is the angular frequency, $\varepsilon_{1}^{T}(\omega)\left[=\varepsilon_{1}^{\Gamma}(\omega)+\varepsilon_{1}^{X, L}(\omega)\right]$ is the real part of the total dielectric function, and $\varepsilon_{2}^{\Gamma}(\omega)$ [= $\left.\varepsilon_{2}^{e x c}(\omega)+\varepsilon_{2}^{b o u n d}(\omega)+\varepsilon_{2}^{c o n}(\omega)\right]$ is the imaginary part of the dielectric function for the $\Gamma$ valley.

The real part of the dielectric function, $\varepsilon_{1}^{\Gamma}(\omega)$, for the $\Gamma$ valley is given by [12]

$$
\begin{aligned}
\varepsilon_{1}^{\Gamma}(\omega)= & +\frac{1}{\pi} \int_{0}^{\infty} \frac{\varepsilon_{2}^{\Gamma}\left(\omega^{\prime}\right)}{\omega^{\prime}+\omega} d \omega^{\prime} \\
& +\frac{1}{\pi} \sum_{m=1}^{M} \int_{\omega_{m}^{\prime}}^{\omega_{m+1}^{\prime}} \frac{\varepsilon_{2}^{\Gamma}\left(\omega^{\prime}\right)}{\omega^{\prime}-\omega} d \omega^{\prime}, \quad \omega_{m}^{\prime} \neq \omega .
\end{aligned}
$$

The imaginary part of the dielectric function for the $\Gamma$ valley, $\varepsilon_{2}^{\Gamma}(\omega)$, is obtained by summing over all the above contributions as follows:

$$
\varepsilon_{2}^{\Gamma}(\omega)=\varepsilon_{2}^{\text {exc }}(\omega)+\varepsilon_{2}^{\text {bound }}(\omega)+\varepsilon_{2}^{\text {con }}(\omega)
$$

where $\varepsilon_{2}^{\mathrm{exc}}(\omega)$ is the $1 \mathrm{~S}$ exciton contribution derived by the density-matrix approach at the subband edge without the influence of band mixing and $\varepsilon_{2}^{\text {bound }}(\omega)$ is the conductionvalence band bound-state contribution without the electronhole interaction. $\varepsilon_{2}^{\operatorname{con}}(\omega)$ is the contribution from the unbound continuum states above the barrier, which are determined using a wider (2000- $\AA$ width) square QW above the DFQW and by the same method for the bound states.

Using the density matrix approach, the optical gain with the photon generated in the direction perpendicular to the surface of QW layers is given as [14]

$$
\begin{aligned}
G(\omega)= & \frac{e^{2} M_{b}^{2}}{\pi c \varepsilon m_{o}^{2} \omega L_{z}} \sum_{p, q} \int\left|\left\langle\psi_{C p} \mid \psi_{V q}\right\rangle\right|^{2} \mathbf{P}_{p q}(k) \\
& \cdot \mathbf{L}\left[E_{p}(k)-E_{q}(k)-\hbar \omega\right] \\
& \cdot\left\{f^{C}\left[E_{p}(k)\right]-f^{V}\left[E_{q}(k)\right]\right\} d k
\end{aligned}
$$

where $m_{o}$ is the rest mass of electron and $M_{b}$ is the optical matrix. $E_{p}$ and $E_{q}$ are the $p$ th-electron and $q$ th-hole subbandedge energy, respectively, and $\psi_{C}$ and $\psi_{V}$ are the envelope 
TABLE III

Material Parameters in the Laser Structure

\begin{tabular}{c|c|c|c|c}
\cline { 2 - 4 } (at operating wavelength of & \multicolumn{4}{|c}{ Diffusion Length $\left(\mathrm{L}_{\mathrm{d}}\right)$} \\
\cline { 2 - 5 } $0.85 \mu \mathrm{m})$ & $50 \AA$ & $60 \AA$ & $80 \AA$ & $100 \AA$ \\
\hline Fitted parameter $\left(\mathrm{a}_{\mathrm{g}}\right) \times 10^{-18} \mathrm{~cm}^{-1}$ & 4.3819 & 4.1967 & 7.0395 & 7.1861 \\
\hline Fitted parameter $\left(\mathrm{N}_{\mathrm{g}}\right) \times 10^{18} \mathrm{~cm}^{-3}$ & 29.8423 & 32.3840 & 27.3708 & 29.6957 \\
\hline Refractive index $\left(\mathrm{n}_{\mathrm{r}}\right)$ & 3.4750 & 3.4690 & 3.4590 & 3.4280 \\
\hline Effective refractive index $(\mathrm{ne})$ & 3.290989 & 3.193064 & 3.181995 & 3.178748 \\
\hline
\end{tabular}

wavefunctions for the electrons and holes, respectively. $\mathbf{L}$ is the Lorentzian broadening factor with HWHM of $5 \mathrm{meV}$ and $k$ is the wavevector. The summation in (B4) is over all the conduction and valence subbands and $\mathbf{P}(k)$ is the TEpolarization factor. $f^{C}$ and $f^{V}$ are the quasi-Fermi for the electrons in the conduction and valance bands, respectively.

Using (B4), the TE optical gain, $G$, at $0.85 \mu \mathrm{m}$ with $L_{d}$ varying between 0 and $20 \AA$ is approximated by

$$
G=a_{o}\left(1+a_{1} T+a_{2} T^{2}\right) \ln \left[\frac{N}{N_{o}\left(1+b_{1} T\right)}\right]
$$

where $T(\geq 300 \mathrm{~K})$ is the temperature, the parameters $a_{o}$, and $N_{o}$ are the gain coefficient and carrier concentration at transparency. In (B5), $a_{O}$ and $N_{o}$ are assumed to be varied with $L_{d}$ and their dependences with $L_{d}$ are given in Table II. The temperature dependence of $G$ is approximated by the coefficients $a_{1}=3.0815 \times 10^{-3} \mathrm{~K}^{-1}, a_{2}=5.31695$ $\times 10^{-6} \mathrm{~K}^{-2}$, and $b_{1}=6.017 \times 10^{-3} \mathrm{~K}^{-1}$. However for $L_{d}>20 \AA$, the TE optical gain, $G$, at $0.85 \mu \mathrm{m}$ is approximated by a linear relation and is given as

$$
G=a_{g}\left(N-N_{g}\right)
$$

where $a_{g}$ and $N_{g}$ are the fitted parameters and are assumed to be varied with $L_{d}>20 \AA$ only. The values of these parameters are given in Table III. The temperature dependence of $G$ with $L_{d}>20 \AA$ is ignored in our calculation because the change of optical loss with operation wavelength is almost negligible (i.e., shift of gain spectrum due to temperature effect) [see Fig. 2(b)].

The carrier-induced refractive index change, $\Delta n$, which varies with the background refractive index profile of the active region, can be obtained from the change of the gain coefficient, $\Delta G(\omega)=G(\omega)-G_{o}(\omega)$, through the Kramers-Kronig dispersion relation [13]

$$
\Delta n(\omega)=\frac{\pi}{c} \mathrm{PV} \int_{0}^{\infty} \frac{\Delta G\left(\omega^{\prime}\right)}{\omega^{\prime 2}-\omega^{2}} d \omega^{\prime}
$$

where $G_{o}(\omega)$ is the optical gain at transparency. The symbol PV stands for the Cauchy principle value. We can also approximate the carrier-induced refractive index change, $\Delta n$, at $0.85 \mu \mathrm{m}$ with $L_{d}$ varying between 0 and $20 \AA$ by the following expression:

$$
\Delta n=d_{0}\left(1+d_{1} T+d_{2} T^{2}\right) \ln \left[\frac{N}{N_{r}\left(1+e_{1} T\right)}\right]
$$

where the parameters $d_{o}$ and $N_{r}$ are assumed to be varied with $L_{d}$ only. Their dependencies on $L_{d}$ are also given in Table II. The temperature dependence of $\Delta n$ is approximated by the coefficients $d_{1}=2.0791 \times 10^{-4} \mathrm{~K}^{-1}, d_{2}=1.3658 \times$ $10^{-7} \mathrm{~K}^{-2}$, and $e_{1}=3.62 \times 10^{-2} \mathrm{~K}^{-1}$. However for $L_{d}>20$ $\AA$, the refractive index change due to the injection carrier concentration and the variation of temperature are ignored in our calculation for the same reason as discussed before.

\section{APPENDIX C}

Fig. 7 shows the periodic DFQW structure of the semiconductor FP laser. The period of the periodic DFQW structure consists of two sections: a gain section $(G)$ with $L_{d}=0 \AA$ and a loss section $(L)$ with $L_{d}=5-100 \AA$. The sequence of the gain/loss section of the periodic DFQW structure is assumed to be $G, L, \cdots, G, L, G, G, L, G, \cdots, L, G$. The neighboring gain sections in the device center are used to provide a $\lambda / 4$ phase-shifted for the excitation of the gap mode. The gain/loss section of the periodic DFQW structure can be represented by a scattering matrix, $M_{k}$, which is given $(\mathrm{C} 1)$, shown at the bottom of the page [17], where $k$ is the section number, $n e$ is the effective refractive index, $\gamma_{k}=j \beta_{k}-g_{k} / 2, \beta_{k}$ (= $\left.2 \pi n e_{k} / \lambda_{o}\right)$ is the propagation constant, and $g_{k}\left(=\Gamma_{k} G_{k}-\alpha_{s}\right)$ is the net power gain of the $k$ th diffused section. $\Delta z_{k}$ in (C1) is the longitudinal length of the diffused sections. The spontaneous emission is exploited in the center of the laser (see Fig. 7) so that the longitudinal propagating fields, $F$ and $R$, can be solved in a matrix format [18] as follows:

$$
\begin{aligned}
{\left[\begin{array}{l}
F_{n}^{\prime} \\
R_{n}^{\prime}
\end{array}\right] } & =\prod_{k=1}^{N_{d}}\left[M_{k}\right]\left[\begin{array}{l}
F_{1} \\
R_{1}
\end{array}\right]+\prod_{k=1}^{N_{d} / 2}\left[M_{k}\right]\left[\begin{array}{c}
I_{f} \\
I_{r}
\end{array}\right] \\
& =\left[\begin{array}{ll}
T_{11} & T_{12} \\
T_{21} & T_{22}
\end{array}\right]\left[\begin{array}{l}
F_{1} \\
R_{1}
\end{array}\right]+\left[\begin{array}{ll}
S_{11} & S_{12} \\
S_{21} & S_{22}
\end{array}\right]\left[\begin{array}{c}
I_{f} \\
I_{r}
\end{array}\right]
\end{aligned}
$$

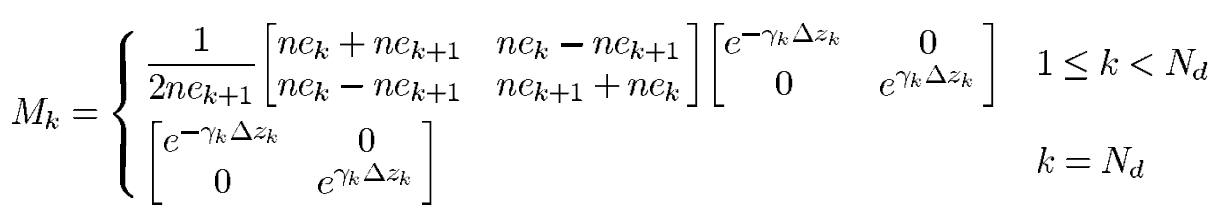


where $I_{f}$ and $I_{r}$ are the spontaneous emission noise coupled into the forward and reverse fields. The lasing conditions for the longitudinal modes can be evaluated from the boundary conditions at the laser facets

$$
F_{1}=r_{L} R_{1}
$$

and

$$
R_{n}^{\prime}=r_{R} F_{n}^{\prime}
$$

where $r_{L}$ and $r_{R}$ are the left and right facet reflectivities, respectively. By substituting $(\mathrm{C} 3)$ into $(\mathrm{C} 2)$, we get

$$
\begin{aligned}
{\left[\begin{array}{c}
r_{L} R_{1} \\
R_{1}
\end{array}\right]=} & {\left[\begin{array}{cc}
0 & 0 \\
r_{L} & 1
\end{array}\right]\left[\begin{array}{cc}
1 & -r_{L} T_{11}-T_{12} \\
r_{R} & -r_{L} T_{21}-T_{22}
\end{array}\right]^{-1} } \\
& \cdot\left[\begin{array}{ll}
S_{11} & S_{12} \\
S_{21} & S_{22}
\end{array}\right]\left[\begin{array}{c}
I_{f} \\
I_{r}
\end{array}\right]
\end{aligned}
$$

and the average photon density, $P$, output from the left facet is given by

$$
P=\left[\begin{array}{rr}
r_{L}^{*} R_{1}^{*} & R_{1}^{*}
\end{array}\right]\left[\begin{array}{c}
r_{L} R_{1} \\
R_{1}
\end{array}\right]
$$

where we have assumed $I_{f} I_{f}^{*}=I_{r} I_{r}^{*}=\nu_{g} B_{s p} \bar{N}^{2} / \Delta z$ and $\bar{N}$ is the average carrier concentration.

\section{ACKNOWLEDGMENT}

The authors would like to thank Dr. P. Shum of the Department of Electrical and Electronic Engineering, Hong Kong University, Hong Kong, or his careful reading of this manuscript.

\section{REFERENCES}

[1] J. S. Major, S. O'Brien, V. Gulgazov, D. F. Welch, and R. J. Lang, "High-power single-mode AlGaAs distributed Bragg reflector laser diodes operating at 856 nm," Electron. Lett., vol. 30, pp. 496-497, 1994

[2] H. Soda, Y. Kotaki, H. Sudo, H. Ishikawa, S. Yamakoshi, and H. Imai, "Stability in single longitudinal mode operation in GaInAsP/InP phaseadjusted DFB lasers," IEEE J. Quantum Electron., vol. QE-23, pp. 804-814, 1987.

[3] J. E. A. Whiteaway, G. H. B. Thompson, A. J. Collar, and C. J. Armistead, "The design and assessment of $\lambda / 4$ phase-shifted DFB laser structure," IEEE J. Quantum Electron., vol. 25, pp. 1261-1279, 1989.

[4] H. Hillmer, K. Magari, and Y. Suzuki, "Chirped gratings for DFB laser diodes using bent waveguides," IEEE Photon. Technol. Lett., vol. 5, pp. $10-12,1993$.

[5] P. Zhou and G. S. Lee, "Chirped grating $\lambda / 4$ shifted DFB laser with uniform longitudinal field distribution," Electron. Lett., vol. 26, pp. 1660-1661, 1990.

[6] J. Salzman, H. Olesen, A. Møller-Larsen, O. Albrektsen, J. Hanberg, J. Nørregaard, B. Jonsson, and B. Tromborg, "Distributed feedback lasers with an S-bent waveguide for high power single mode operation," IEEE J. Select. Topics Quantum Electron., vol. 1, pp. 346-355, 1996.

[7] S. Hansmann, H. Hillmer, H. Walter, H. Burkhard, B. Hubner, and E. Kuphal, "Variation of coupled coefficients by sampled gratings in complex coupled distributed feedback lasers," IEEE J. Select. Topics Quantum Electron., vol. 1, pp. 341-345, 1996.

[8] G. P. Agrawal and N. K. Dutta, Long-Wavelength Semiconductor Lasers. New York: Van Nostrand Reinhold, 1986.

[9] C. C. Lee and D. H. Chien, "The effect of bonding wires on longitudinal temperature profiles of laser diode," J. Lightwave Technol., vol. 30, vol. 14, pp. 1847-1852, 1996.

[10] J. Kinoshita, K. Ohtsuka, H. Agatsuma, A. Tanaka, T. Matsuyama, A. Makuta, and H. Kobayashi, "Performance of $1.5 \mu \mathrm{m}$ DFB lasers with a narrow stripe region," IEEE J. Quantum Electron., vol. 27, pp. 1759-1765, 1991.
[11] L. M. Zhang, S. F. Yu, M. C. Nowell, D. D. Marcenac, J. E. Carroll, and R. G. S. Plumb, "Dynamic analysis of radiation and side-mode suppression in a second-order DFB laser using time domain large signal travelling wave model," IEEE J. Quantum Electron., vol. 30, pp. 1389-1395, 1994.

[12] E. H. Li, B. L. Weiss, K. S. Chan, and J. Micallef, "The polarization dependent refractive index of an interdiffusion induced $\mathrm{AlGaAs} / \mathrm{GaAs}$ quantum well," Appl. Phys. Lett., vol. 62, pp. 550-552, 1992.

[13] C. H. Herny, R. A. Logan, and K. A. Bertness, "Spectral dependence of the change in refractive index due to carrier injection in GaAs lasers," J. Appl. Phys., vol. 52, pp. 4457-4461, 1981.

[14] E. H. Li and K. S. Chan, "Laser gain and current density in a disordered AlGaAs/GaAs quantum well," Electron. Lett., vol. 29, pp. 1233-1234, 1993.

[15] W. P. Gillin, I. V. Bradley, L. K. Howard, R. Gwilliam, and K. P. Homewood, "The effects of silicon and beryllium on the interdiffusion of GaAs/Al $\mathrm{Al}_{x} \mathrm{Ga}_{1-x} \mathrm{As} / \mathrm{GaAs}$ quantum well structures," J. Appl. Phys., vol. 73, no. 11, pp. 7715-7719, 1993.

[16] R. F. Kazarinov and C. H. Henry, "Second-order distributed feedback lasers with mode selection provided by first-order radiation loss," IEEE J. Quantum Electron., vol. QE-21, pp. 144-150, 1985.

[17] E. Hecht, Optics. Boston, MA: Addison-Wesley, 1974, ch. 4.

[18] L. M. Zhang and J. E. Carroll, "Large signal dynamic model of the DFB lasers," IEEE Quantum Electron., vol. 28, pp. 604-611, 1992.

[19] S. R. Andrew, J. H. Marsh, M. C. Holland, and A. H. Kean, "Quantum well laser with integrated passive waveguide fabrication by neutral impurity disordering," IEEE Photon. Technol. Lett., vol. 4, pp. 426-428, 1992.

[20] C. Kaden, H. Grabeldinger, H. P. Gauggel, V. Hofsa $\beta$, A. Hase, A. Menschig, H. Schweizer, R. Zengerle, and H. J. Bruckner, Microelectron. Eng., vol. 23, p. 469, 1994.

[21] S. F. Yu and E. H. Li, "Proposed enhancement of side mode suppression ratio in $\lambda / 4$ shifted distributed feedback lasers with nonuniform diffused quantum wells," IEEE Photon. Technol. Lett., vol. 8, pp. 482-484, 1996.

[22] V. Hofsäß, J. Kuhn, C. Kaden, V. Härle, H. Bolay, F. Scholz, H. Schweizer, H. Hillmer, R. Lösch, and W. Schlapp, "Optical integration of laterally modified multiple quantum well structures by implantation enhanced intermixing to realize gain coupled DFB lasers," Nucl. Instrum. Methods Phys. Res. B, vol. 106, pp. 471-476, 1995.

[23] W. B. Joyce and R. W. Dixon, "Thermal resistance of heterstructure lasers," J. Appl. Phys., vol. 46, pp. 855-862, 1975.

[24] R. S. Zory, Ed. Quantum Well Lasers. New York: Academic, 1993, ch. 1 .

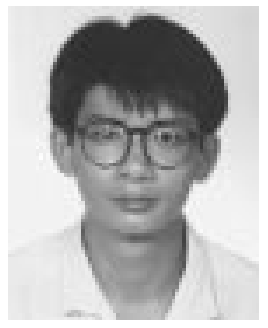

S. F. Yu received the B.Eng. degree (with Departmental Prize) in electronic engineering from London University, University College, England, in 1990 and the Ph.D. degree in optoelectronics from Cambridge University, Robinson College, England, in1993.

He joined the Department of Electronic Engineering, Sha Tin Technical Institute, Hong Kong, as a part-time lecturer in 1993. In 1994, he joined the Department of Electrical and Electronic Engineering, the University of Hong Kong, Hong Kong, where he was a Lecturer. since 1996, he has been an Assistant Professor in the same department. His main research topics include wavelength selectivity of grating coupled waveguides, transient properties of semiconductor laser diodes, and design of optoelectronics integrated circuit. He currently conducts the development of high-performance semiconductor lasers using diffused quantum-well material for the application in high-speed communication systems. He is also involved in the investigation of optical soliton fiber communication systems. He has published over 40 technical papers and one book chapter

Dr. Yu is a Fellow and Honorary Scholar of the Cambridge Commonwealth Trust Society. He held a Croucher Foundation scholarship and an overseas research student award while completing the doctoral program. His biography is published in the fourth edition of Who's Who in Science and Engineering, the 25th edition of The Dictionary of International Biographies, an the 17th edition of The International Directory of Distinguished Leadership. For further information, contact the personal home page at http://www.eee.hku.hk/ sfyu. 


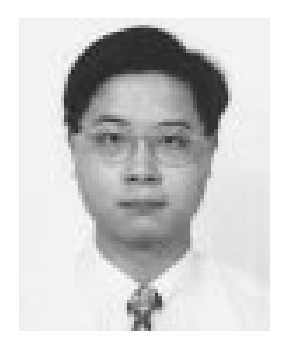

C. W. Lo was born in Hong Kong on October 29, 1971. He received the B.Sc. (Honors) degree in physics from the University of Hong Kong in 1994. He is currently working towards the M.Phil. degree at the same institution and will be graduating this year. His thesis is titled "Improvement of Semiconductor Laser Diodes' Characteristics by Using Diffused Quantum Wells Structure."

His research interest are concerned with the development of high-performance semiconductor lasers (including Fabry-Perot, distributed feedback, and vertical-cavity surface-emitting types) using diffused quantum-well structure. The influence of spatial hole burning of carrier concentration, thermal effects, carrier transport, waveguide dimension, and other nonlinear optical properties of semiconductor lasers are also considered in the investigation. He has published six technical papers and one book chapter while pursuing the M.Phil degree.

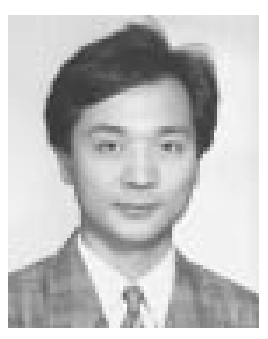

E. Herbert Li (S'87-M'88-SM'95) received the M.Phil. degree in applied mathematics and electronic engineering from the University of Hong Kong and the Ph.D. degree in electronic engineering from the University of Surrey, U.K.

$\mathrm{He}$ worked for the Kirsten Aeronautical Laboratory, Seattle, WA (1979-1980). He joined K $\mathrm{K}$ Engineering Co., Hong Kong, as an Engineer (1981-1986), and Micro Systems, Hong Kong, as a Manager (1986-1988). He was a Member of Faculty at the City University of Hong Kong (1988-1990). $\mathrm{He}$ joined the National Ion Implantation Facility at the Department of Electrical and Electronic Engineering, University of Surrey (1990-1993). He is currently a Member of Faculty and Leader of the Optoelectronics Group, which consists of a team of 10 researchers, at the Department of Electrical and Electronic Engineering, University of Hong Kong (1994-present). $\mathrm{He}$ is an Adjunct Professor of the Institute of Semiconductors, Shandong Normal University, China, and a Visiting Faculty Member at the University of Waterloo, Canada. His current research interests are mainly concerned with optoelectronic device fabrication, modeling and characterization, and in particular interdiffusion induced modification of quantum-well structures for the advanced performance and integration of optoelectronic devices. Since 1990, he has published over 100 international technical papers, two book chapters, and three books in the above area. He serves on both the Technical Program and International Advisory Committees of OECC in 1996 (Japan) and 1997 (Korea). He is an Editor of the International Journal of Optoelectronics.

Dr. $\mathrm{Li}$ is a "Distinguished Lecturer" of IEEE-EDS for 1997, and he is an AdCom ex officio member and EDS Meeting Committee member of the IEEE. He received the J. Langham Thompson Premium Prize in 1992 from the Institution of Electrical Engineers (U.K.) and the Distinguished Pioneering Projects Award in 1989 from HKCSS (Hong Kong). His biography is published in Who's Who in Science and Engineering. 